\title{
Haar wavelet method for solving coupled system of fractional order partial differential equations
}

\author{
Abbas AL-Shimmary ${ }^{1}$, Sajeda Kareem Radhi ${ }^{2}$, Amina Kassim Hussain ${ }^{3}$ \\ ${ }^{1}$ Department of Electrical Engineering, Faculty of Engineering, University of Al-Mustansiriyah, Baghdad, Iraq \\ ${ }^{2}$ Department of Remote Sensing, College of Remote Sensing \& Geophysics, AL-Karkh University, Baghdad, Iraq \\ ${ }^{3}$ Department of Meterial Engineering, Faculty of Engineering, University of Al-Mustansiriyah, Baghdad, Iraq
}

\section{Article Info}

Article history:

Received Jun 5, 2020

Revised Sep 18, 2020

Accepted Nov 30, 2020

\section{Keywords:}

Fractional order partial

Differential equations

Haar wavelet

\begin{abstract}
This paper deal with the numerical method, based on the operational matrices of the Haar wavelet orthonormal functions approach to approximate solutions to a class of coupled systems of time-fractional order partial differential equations (FPDEs.). By introducing the fractional derivative of the Caputo sense, to avoid the tedious calculations and to promote the study of wavelets to beginners, we use the integration property of this method with the aid of the aforesaid orthogonal matrices which convert the coupled system under some consideration into an easily algebraic system of Lyapunov or Sylvester equation type. The advantage of the present method, including the simple computation, computer-oriented, which requires less space to store, timeefficient, and it can be applied for solving integer (fractional) order partial differential equations. Some specific and illustrating examples have been given; figures are used to show the efficiency, as well as the accuracy of the, achieved approximated results. All numerical calculations in this paper have been carried out with MATLAB.
\end{abstract}

This is an open access article under the CC BY-SA license.

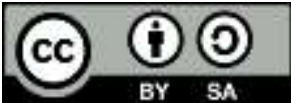

\section{Corresponding Author:}

Abbas Fadhil Al-Shimmary

Department of Electrical Engineering

Al-Mustansiriyah University, Sec.322, St.30, Baghdad, Iraq

Email: abbs.fadhil62@gmail.com

\section{INTRODUCTION}

The subject of fractional calculus is a branch of applied mathematics which deal with derivatives and integrals of any arbitrary orders. In this study, we introduce fractional order partial differential equations (FPDEs) which represent an effective tool to describe several certain real physical phenomena. Most of the engineering and physical processes are naturally led to (FPDEs), such as damping laws, dynamical processes in fluid, and porous structure [1,2]. FPDEs appear also in the control theory of dynamical systems, when the controlled system is delineated, most studies show that, the fractional-order controller can provide better performance than the controller with an integer order. Also, most coupled systems of FPDEs can be found in biomechanics, as the modeling phenomena of electrical activity in the heart [3-5]. Furthermore, it appears in solid-state physics and mechanics, as the coupled system is described by the dynamics of multi-deformable bodies coupled with standard light fractional order discrete continuum layers [6-9]. And since most of FPDEs do not have exact analytic solutions, so the branch dealing with an approximate numerical solution of this type of differential equations has attracted the attention of many researchers in recent years. Some of these methods are; Adomain decomposition method (ADM) [10]. The generalized differentials transform method (GDTM) [11]. Variation iteration method (VIM) [12]. Wavelet method (WM) [13]. Generalized block pulse operational matrix method (GBPM) [14]. The homotopy perturbation method (HPM) and Stehfest's 
numerical algorithm for the calculation of inverse (LT) [15], etc. In this study, we focus on the numerical method of solving a coupled system of the fractional partial differential equation based on the operational matrices of Haar wavelet orthonormal functions.

The main aim of this study is to obtain fast and effective algorithms that are suitable for digital computers. The rest of this paper is organized as follows: In Section 2, we recall some necessary definitions of the fractional calculus theory to make this article self-contained. The Harr wavelet concept has been presented in Section 3. Section 4 is devoted to the operational matrices of the Haar wavelet, illustrating examples of the applied method is introduced in Section 5. Finally, conclusion and future studies are introduced in Section 6.

\section{PRELIMINARIES}

This section is devoted to some fundamental and necessary definitions of the theory of fractional calculus, which are required for building up our outcomes [16, 17].

Definition 1:

A real function $u(x, t), x, t \in R, t>0$ is said to be in the space $\mathbf{C}_{\mu}, \mu \in R$, if there exist a real number $p>\mu$, such that $u(x, t)=t^{p} u_{1}(x, t)$, where $u_{1}(x, t) \in C(R \times[0, \infty))$, and it is said to be in space $\mathrm{C}_{\mu}^{\mathrm{n}}$ if and only if $\frac{\partial^{n} u(x, t)}{\partial t^{n}} \in \mathbf{C}_{\mu}, n \in \mathbf{N} \cup\{\mathbf{0}\}$.

Definition 2:

The (left sided) Riemann-Liouville fractional integral operator $\left(j^{\alpha}\right)$ of order $\alpha>\mathbf{0}$, of a function $u(x, t) \in \mathbf{C}_{\mu}, \mu \geq-1$, with respect to $\mathrm{t}$ is defined as:

$$
\left\{\begin{array}{l}
j_{t}^{\alpha} u(x, t)=\frac{1}{\Gamma(\alpha)} \int_{0}^{t}(t-\tau)^{\alpha-1} u(x, \tau) d \tau, \quad t>0 \\
j_{t}^{0} u(x, t)=u(x, t)
\end{array}\right.
$$

Definition 3:

The (left sided) fractional derivative operator ${ }^{c} D_{t}^{\alpha}$ of order $\alpha \geq 0$, of a function $u(x, t) \in \mathbf{C}_{\mu}, \mu \geq-1$, with respect to "t" in the Caputo sense is defined as, see [18]:

$$
{ }^{c} D_{t}^{\alpha} u(x, t)= \begin{cases}\frac{1}{\Gamma(n-\alpha)} \int_{0}^{t} \frac{\frac{\partial^{n} u(x, \tau)}{\partial t^{n}}}{(t-\tau)^{\alpha-n+1}} d \tau, & 0 \leq n-1<\alpha<n, \\ \frac{\partial^{n}}{\partial t^{n}} u(x, t), & \alpha=n .\end{cases}
$$

Where $n-1<\alpha \leq n, n \in \mathrm{N}, n=\lceil\alpha\rceil$ and $\lceil\alpha\rceil$ is the greatest positive integer function. The fractional derivative of order $\alpha$ in the sense of Caputo is also defined as.

${ }^{c} D_{t}^{\alpha} u(x, t)=j^{\alpha-r} D_{t}^{r} u(x, t)$, where $D_{t}^{r}$ is the usual differential operator of order $\mathrm{r}$. The relation between Caputo differential operator and Riemann - Liouville integral operator is given by the expression:

$$
\begin{aligned}
& D_{t}^{\alpha} j_{t}^{\alpha} u(x, t)=u(x, t), \\
& j_{t}^{\alpha} D_{t}^{\alpha} u(x, t)=u(x, t)-\sum_{k=0}^{n-1} \frac{\partial^{k} u\left(x, 0^{+}\right)}{\partial t^{k}} \frac{t^{k}}{k !}, \quad t>0 .
\end{aligned}
$$




\section{HAAR WAVELETS}

A wavelet is a mathematical function used in signal processing and image compression, in the first applied in geophysics to get pictures of layers in subsurface rock, which are used in oil and mineral exploration. One of the popular families of wavelets is the Haar wavelet, it is the oldest wavelet, it was introduced by the Hungarian mathematician Alfred Haar in 1909, and the wavelets theory started much in the 1980s. It is a popular topic in diverse fields of engineering and science, the Haar wavelet is also considered the first known simplest wavelet because it was consisting of a piecewise constant function, that take only the three values 0,1 and -1 . In the last two decades, the subject of wavelets theory has been playing an important role in many computing mathematical scientists, especially in time-frequency analysis, signal analysis, and numerical analysis, wavelet technique enables us to decomposed different complicated functions into a summation of basic functions, as well as each basic function can be generated from dilation and translation of a mother wavelet function. Consequently, the wavelet analysis of functions is more accurately described and faster than Fourier analysis. Many studies are focusing on the method of orthogonal polynomial and functions, to obtain an effective algorithm that can be suitable for adigital computer. Such as anumerical method with omitting discretization [19], Haar wavelet functions [20-24], etc.

In this work, we focus on the Haar wavelet method based on the orthogonal matrix including the integration of orthonormal functions, without using Haar wavelet-based on block pulse functions that differ from it, are utilized to reduce the fractional partial differential equation into an easily algebraic matrix equation one of Lyapunov or Sylvester type, which can be solved by computer oriented methods, the calculations are done by using Math lab software with certain commands or special toolbox. Moreover, it is an effective tool in the computational sciences, especially in the subject of signal analysis, data compression, and many others, and because its features, such as high energy packing, flexible, the non-stability of numerical solutions does not occur in this method, effective for treating singularities, moreover small computational cost and it has properties that help speed calculations, after this introduction. Let us begin with the definition of the orthonormal set of Haar functions, see [25], which is a family of switched rectangle waveform with amplitude differ one to another, it contains just one wavelet during the interest unit subinterval of time $[0,1)$, remain zero elsewhere, and orthonormal, the $\mathrm{i}^{\text {th }}$ uniform Haar wavelet $h_{i j}(t)$ is define as:

$$
\begin{aligned}
& h_{\mathrm{oo}}(t)=\frac{1}{\sqrt{m}} \begin{cases}1, & 0 \leq t<1, \\
0, & \text { otherwise. }\end{cases} \\
& h_{i j}(t)=\frac{1}{\sqrt{m}}\left\{\begin{array}{cc}
2^{j / 2}, & t \in\left[\frac{k-1}{2^{j}}, \frac{k-0.5}{2^{j}}\right), \\
-2^{j / 2}, & t \in\left[\frac{k-0.5}{2^{j}}, \frac{k}{2^{j}}\right), \\
0, & \text { otherwize. }
\end{array}\right.
\end{aligned}
$$

Where the dilation parameter $j=0,1,2, \ldots, J, m=2^{j+1}$, and $\mathrm{J}$ is the maximum level of resolution, the parameter $k=1,2, \ldots, m-1$ represents the translation of the wavelet, the integers $k, j$ represent the decomposition of the index $i, i=2^{j}+k-1$, the maximum value of $i$ is $i=2 M=2^{J+1}$, there are two functions play aprimary role in wavelet analysis, the function $h_{00}(t)$, which is called a Haar father function and the function $h_{10}(t)$, which is called a Haar mother function that generates whole other daughter and granddaughter functions on unit interval $[0,1)$, there are two basic characteristics occurs in Haar functions represent by translation (shifting) and dilation, while the rest of functions can be generated from $h_{10}(t)$ using these two operations and knowledge by the following relationship:

$$
h_{i j}(t)=h_{1 j}\left(2^{j} t-k / 2^{j}\right), i=2^{j}+k-1, j \geq 0
$$
and satisfies:

Each Haar wavelet consists of a couple of constant steps with an opposite sign during its subinterval 


$$
\int_{0}^{1} h_{i j}(t) h_{r j}(t) d t= \begin{cases}2^{-j}, & i=r, \\ \mathbf{0}, & i \neq r .\end{cases}
$$

This relationship shows that Haar wavelets are orthonormal therefore, it forms an orthonormal basis, and in this case, we have an explicit formula for the unique coefficients in the linear combination. With the dilation and translation process, one can easily obtain basis functions of two dimensions, as shown in Figure 1.

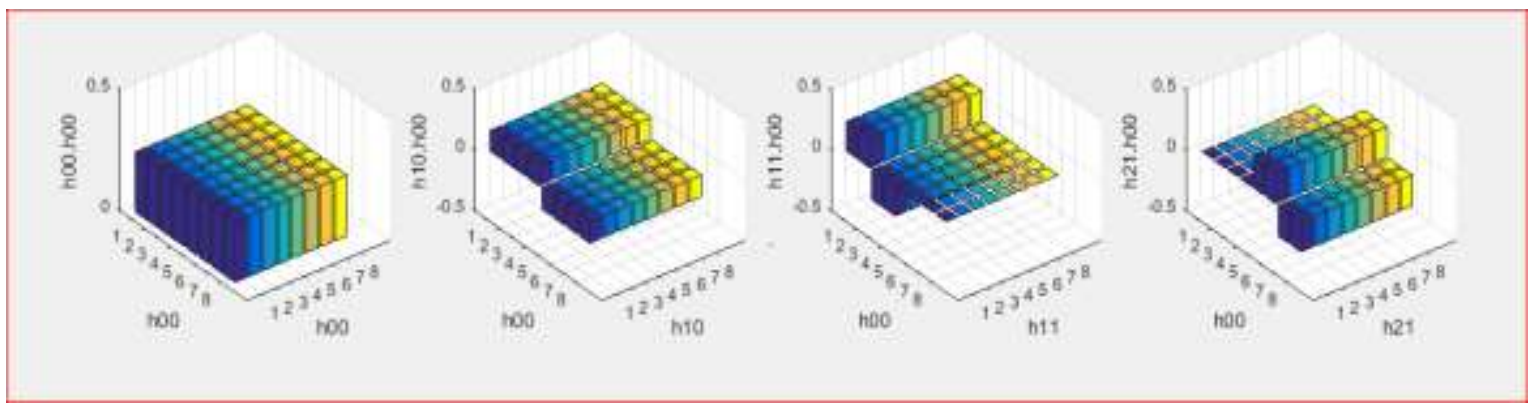

Figure 1. The first 1x4 basis for Haar wavelet orthogonal functions with $m=8$

In this case, the Haar orthonormal matrix for resolution up to 2 levels is given by:

$$
H_{8 \times 8}(t)=\left[\begin{array}{l}
h_{\mathrm{OO}}(t) \\
h_{10}(t) \\
h_{20}(t) \\
h_{21}(t) \\
h_{30}(t) \\
h_{31}(t) \\
h_{32}(t) \\
h_{33}(t)
\end{array}\right]=\left[\begin{array}{cccccccc}
\frac{1}{\sqrt{8}} & \frac{1}{\sqrt{8}} & \frac{1}{\sqrt{8}} & \frac{1}{\sqrt{8}} & \frac{1}{\sqrt{8}} & \frac{1}{\sqrt{8}} & \frac{1}{\sqrt{8}} & \frac{1}{\sqrt{8}} \\
\frac{1}{\sqrt{8}} & \frac{1}{\sqrt{8}} & \frac{1}{\sqrt{8}} & \frac{1}{\sqrt{8}} & \frac{-1}{\sqrt{8}} & \frac{-1}{\sqrt{8}} & \frac{-1}{\sqrt{8}} & \frac{-1}{\sqrt{8}} \\
\frac{1}{2} & \frac{1}{2} & \frac{-1}{2} & \frac{-1}{2} & 0 & 0 & 0 & 0 \\
0 & 0 & 0 & 0 & \frac{1}{2} & \frac{1}{2} & \frac{-1}{2} & \frac{-1}{2} \\
\frac{1}{\sqrt{2}} & \frac{-1}{\sqrt{2}} & 0 & 0 & 0 & 0 & 0 & 0 \\
0 & 0 & \frac{1}{\sqrt{2}} & \frac{-1}{\sqrt{2}} & 0 & 0 & 0 & 0 \\
0 & 0 & 0 & 0 & \frac{1}{\sqrt{2}} & \frac{-1}{\sqrt{2}} & 0 & 0 \\
0 & 0 & 0 & 0 & 0 & 0 & \frac{1}{\sqrt{2}} & \frac{-1}{\sqrt{2}}
\end{array}\right],
$$

Where

$$
\begin{aligned}
& \mathrm{H}_{8}\left(\frac{1}{16}\right)=\left[\begin{array}{llllllll}
\frac{1}{\sqrt{8}} & \frac{1}{\sqrt{8}} & \frac{1}{2} & 0 & \frac{1}{\sqrt{2}} & 0 & 0 & 0
\end{array}\right]^{T}, \\
& \mathrm{H}_{8}\left(\frac{3}{16}\right)=\left[\begin{array}{llllllll}
\frac{1}{\sqrt{8}} & \frac{1}{\sqrt{8}} & \frac{1}{2} & 0 & \frac{-1}{\sqrt{2}} & 0 & 0 & 0
\end{array}\right]^{T}, \\
& \mathbf{H}_{8}\left(\frac{15}{16}\right)=\left[\begin{array}{llllllll}
\frac{1}{\sqrt{8}} & \frac{1}{\sqrt{8}} & \mathbf{0} & \frac{1}{2} & \mathbf{0} & \mathbf{0} & \mathbf{0} & \frac{-\mathbf{1}}{\sqrt{2}}
\end{array}\right]^{T} .
\end{aligned}
$$

\section{THE OPERATIONAL MATRICES OF HAAR WAVELET}

In this section, we introduce the operational matrices of the Haar wavelet. The main idea of an operational matrix is utilized to reduce the integer (fractional) ordinary (partial) differential equation into an algebraic simple matrix equation of Lyapunov (Sylvester) type, which can be solved easily by a computeroriented method. 


\subsection{Function approximation}

Now, similar as one-dimensional function, any two-dimensional function $u(x, t) \in$ $L^{2}([0,1) \times[0,1))$ which is a piecewise constant or approximately piecewise constant during each subinterval and square integrable in the intervals $0 \leq x<1$ and $0 \leq t<1$ also can be decomposed into an infinite Haar series as see [26]:

$$
u(x, t)=\sum_{i=1}^{\infty} \sum_{j=1}^{\infty} c_{i, j} h_{i}(x) h_{j}(t)
$$

Where $c_{i, j}$ is $2 M \times 2 M$ coefficient matrix, which can be determined from the inner product:

$$
\begin{aligned}
c_{i, j} & =\left\langle h_{i}(x),\left\langle u(x, t), h_{j}(t)\right\rangle\right\rangle, \\
& =\mathbf{2}^{\mathbf{2 j}} \int_{0}^{1} u(x, t) h_{i}(x) d x \int_{0}^{1} u(x, t) h_{j}(t) d t .
\end{aligned}
$$

And called the wavelet coefficients. Let $H_{m}(x)=\left[h_{0}(x), h_{1}(x), \cdots, h_{m-1}(x)\right]^{T}$ and $H_{m}(t)=\left[h_{0}(t), h_{1}(t), \cdots, h_{m-1}(t)\right]^{T}$, here $H_{m}$ is called the Haar wavelet matrix of order $\mathrm{m}$, where $m=2^{j}$ as previously mentioned, and $T$ denotes the transpose of the matrix, hence the approximated sum of $m$ terms of the finite series of (10) can be written in matrix form as:

$$
u(x, t) \cong \sum_{i=0}^{m-1} \sum_{j=0}^{m-1} c_{i, j} h_{i}(x) h_{j}(t)
$$

Now, to determine the coefficients $c_{i, j}$, we will apply the wavelet collocation method, these shown in the following:

$$
x_{\ell}=t_{\ell}=(\ell-0.5) / 2 M, \ell=1,2, \cdots, 2 M
$$

Write (12) in discrete form by using (13), we obtain the matrix equation:

$$
U(x, t)=H_{m}^{T}(x) C H_{m}(t)
$$

Where $U=\left[u\left(x_{i}, t_{j}\right)\right]_{m \times m}, C=\left[c_{i, j}\right]_{m \times m}$ and $H_{m}$ is called Haar wavelet matrix of order $m$, i.e:

$$
H_{m}=\left[\begin{array}{c}
\vec{h}_{0} \\
\vec{h}_{1} \\
\vdots \\
\vec{h}_{m-1}
\end{array}\right]=\left[\begin{array}{ccccc}
h_{0,0} & h_{0,1} & h_{0,2} & \cdots & h_{0, m-1} \\
h_{1,0} & h_{1,1} & h_{1,2} & \cdots & h_{1, m-1} \\
\vdots & \vdots & \vdots & \ddots & \vdots \\
h_{m-1,0} & h_{m-1,1} & h_{m-1,2} & \cdots & h_{m-1, m-1}
\end{array}\right],
$$

Here $\overrightarrow{\mathrm{h}}_{0}, \vec{h}_{1}, \vec{h}_{2}, \cdots, \vec{h}_{m-1}$ are the discrete forms of the Haar wavelet basis. Since the Haar wavelet matrix is orthogonal as mentioned earlier (i.e. $H_{m}^{-1}=H_{m}^{T}$ ), accordingly the wavelet coefficients can be calculated from (14) by:

$$
C=H_{m \times m} U H_{m \times m}^{T}
$$




\subsection{Haar wavelet of Integer Order}

To apply the Haar wavelet method for solving any integer order partial differential equations one needs the integrals below as presented in [23]:

$$
\int_{0}^{t} h_{0}(\tau) \mathrm{d} \tau=\frac{1}{\sqrt{\mathrm{m}}} t, \quad 0 \leq t<1
$$

For $i=1,2, \ldots, m-1$, can be described as below:

$$
\int_{0}^{t} h_{i}(\tau) \mathbf{d} \tau=\frac{\mathbf{2}^{j / 2}}{\sqrt{\mathbf{m}}} \begin{cases}t-\frac{k}{2^{j}}, & \frac{k}{2^{j}} \leq t<\frac{k+0.5}{2^{j}}, \\ \frac{k+1}{2^{j}}-t, & \frac{k+0.5}{2^{j}} \leq t<\frac{k+1}{2^{j}}, \\ \mathbf{0} & , \text { otherwise } .\end{cases}
$$

And the matrix form of integration of Haar wavelet is:

$$
\int_{0}^{t} p_{m}^{n-1} H_{m}(\tau) d \tau \cong p_{m}^{n} H_{m}(t), \quad n=1,2,3, \ldots .
$$

Similarly, for integration the transpose Haar wavelet:

$$
\int_{0}^{t} H_{m}^{T}(\tau) d \tau \cong H_{m}^{T}(t)\left(p_{m}^{1}\right)^{T}
$$

Where the $m \times m$ matrix $p_{m}^{1}$ is called the Haar wavelet operational matrix of one-time integration of matrix $H_{m}(t)$, Chen and Hsaio in [23], showed that the matrix $p_{m}^{1}$ can be calculated from the partition four submatrices:

$$
p_{m}^{1}=\frac{1}{2 m}\left[\begin{array}{cc}
2 m P_{\mathrm{m} / 2}^{1} & -H_{m / 2} \\
\hline H_{m / 2}^{-1} & \mathbf{0}
\end{array}\right]
$$

Where 0 is the zero matrix of order $m / 2 \times m / 2$.

\subsection{Haar Wavelet of Fractional Order}

Now, like what we have done with the integer order, we will likewise to derive the Haar wavelet operational matrix of fractional order integration $p_{m}^{\alpha}, \alpha \in R^{+}$. For this purpose, we will recall the Riemann-Liouville fractional integral operator $\left(j^{\alpha}\right)$ using definition (2):

$$
j^{\alpha} H_{m}(t)=p_{m}^{\alpha} H_{m}(t)=\left[p_{m}^{\alpha} h_{0}(t), p_{m}^{\alpha} h_{1}(t), \ldots, p_{m}^{\alpha} h_{m-1}(t)\right]^{T}
$$

Where,

$$
p_{m}^{\alpha} h_{0}(t)=\left\{\begin{array}{c}
\frac{t^{\alpha}}{\sqrt{m} \Gamma(\alpha+1)}, t \in[0,1), \\
\mathbf{0} \text { elsewhere. }
\end{array}\right.
$$


For $i=1,2, \ldots, m-1$

$$
p_{m}^{\alpha} h_{i}(t)=\frac{2^{j / 2}}{\sqrt{m} \Gamma(\alpha+1)}\left\{\begin{array}{lr}
0, & \mathbf{0} \leq \mathbf{t}<\frac{\mathbf{k}-\mathbf{1}}{2^{\mathbf{j}}}, \\
\left(t-\frac{k-1}{2^{j}}\right)^{\alpha}, & \frac{\mathbf{k}-\mathbf{1}}{\mathbf{2}^{\mathbf{j}}} \leq \mathbf{t}<\frac{\mathbf{k}-\mathbf{0 . 5}}{\mathbf{2}^{\mathbf{j}}}, \\
\left(t-\frac{k-1}{2^{j}}\right)^{\alpha}-2\left(t-\frac{k-0.5}{2^{j}}\right)^{\alpha}, & \frac{\mathbf{k}-\mathbf{0 . 5}}{\mathbf{2}^{\mathbf{j}}} \leq \mathbf{t}<\frac{\mathbf{k}}{\mathbf{2}^{\mathbf{j}}}, \\
\left(t-\frac{k-1}{2^{j}}\right)^{\alpha}-2\left(t-\frac{k-0.5}{2^{j}}\right)^{\alpha}+\left(t-\frac{k}{2^{j}}\right)^{\alpha}, \quad \frac{\mathbf{k}}{2^{\mathbf{j}}} \leq \mathbf{t}<1 .
\end{array}\right.
$$

Let $D_{m \times m}^{\alpha}$ denote to the Harr wavelet operational $m \times m$ matrix fractional differentiation of order $\alpha$, since $D_{m \times m}^{\alpha} p_{m \times m}^{\alpha}=I$, then we can calculate $D_{m \times m}^{\alpha}$ by inverting $p_{m \times m}^{\alpha}$. Moreover, the Haar wavelet fractional partial derivatives of order $\alpha$ define by:

$$
\frac{\partial^{\alpha} u(x, t)}{\partial t^{\alpha}}=\frac{\partial^{\alpha} U}{\partial t^{\alpha}}=H_{m}^{T}(x) C\left[\frac{\partial^{\alpha} H_{m}(t)}{\partial t^{\alpha}}\right]=H_{m}^{T}(x) C P_{m}^{-\alpha} H_{m}(t),
$$

And

$$
\frac{\partial^{\beta} u(x, t)}{\partial x^{\beta}}=\frac{\partial^{\beta} U}{\partial x^{\beta}}=\frac{\partial^{\beta} H_{m}^{T}(x)}{\partial x^{\beta}} C H_{m}(t)=H_{m}^{T}(x)\left[P_{m}^{-\beta}\right]^{T} C H_{m}(t) .
$$

As well as, Haar wavelet fractional integration of order $\alpha$ with respect to $t$ and $x$ respectively given by:

$$
\begin{aligned}
& j_{t}^{\alpha} U=j_{t}^{\alpha} H_{m}^{T}(x) C H_{m}(t)=H_{m}^{T}(x) C j_{t}^{\alpha}\left(H_{m}(t)=H_{m}^{T} C p_{m}^{\alpha} H_{m},\right. \\
& j_{x}^{\alpha} U=j_{x}^{\alpha}\left(H_{m}^{T}(x) C H_{m}(t)\right)=j_{x}^{\alpha}\left(H_{m}^{T}(x)\right) C\left(H_{m}(t)=H_{m}^{T}\left(p_{m}^{\alpha}\right)^{T} C H_{m} .\right.
\end{aligned}
$$

\subsection{The Methodology of Harr wavelet}

In this section, we apply the Haar wavelet orthonormal matrix method for approximate solutions of coupled system of fractional partial differential equations. All computations and plots have been done in the help of MATLAB. Consider the coupled system for time dependent fractional partial differential equations of orders $\alpha$ and $\beta$ respectively of the kind:

$$
\begin{aligned}
& D_{t}^{\alpha} u(x, t)+f\left(u, v, u_{x}, v_{t}, u_{x x}\right)=f^{1}(x, t), \\
& D_{t}^{\beta} v(x, t)+g\left(u, v, u_{x}, v_{t}, u_{x x}\right)=f^{2}(x, t), \quad 0<\alpha, \beta<1 .
\end{aligned}
$$

With initial and boundary conditions:

$$
\begin{aligned}
& u(0, t)=\xi(t), v(0, t)=\eta(t), \quad u(x, 0)=\vartheta(x), v(x, 0)=\varphi(x), \\
& u_{x}(0, t)=\lambda(t), v_{x}(0, t)=\mu(t), \ldots
\end{aligned}
$$

Where $f^{1}(x, t), f^{2}(x, t), \xi(t), \eta(t), \vartheta(x), \varphi(x), \lambda(t)$ and $\mu(t)$ are known continuous functions, and $0 \leq x, t<1$. Our method can be summarized as the step by step procedure below:

Step1: Sampling the continuous functions $u(x, t)$ and $v(x, t)$ into discrete matrices form $U=\left[u_{i, j}\right]$ and $V=\left[v_{i, j}\right]$ where $\Delta=1 / 2 m$ of $x$ and $t$.

Step 2: Transfer the matrices into Haar domain by using Haar wavelet transform. 


$$
U=H^{T}(x) A H(t), \quad V=H^{T}(x) B H(t) .
$$

Where $A=\left[a_{i, j}\right], B=\left[b_{i, j}\right]$ are the Haar coefficient matrices and $H$ is the Haar wavelet matrix.

Step 3: Write all integer and fractional partial differentiations in Haar wavelet transform.

Step 4: Calculate the wavelet weighting coefficients A and B after plugging (step 3), with initial and boundary conditions (30) in (29):

$$
\left.\begin{array}{l}
H^{T}(x) A P_{m}^{\alpha} H(t)+u(x, 0)+f\left\{H^{T}(x)\left[A, B,\left(P_{m}^{1}\right)^{T} A, B P_{m}^{1}, \cdots\right] H(t)\right\}=H^{T}(x) F^{1} H(t), \\
H^{T}(x) A P_{m}^{\beta} H(t)+u(x, 0)+f\left\{H^{T}(x)\left[A, B,\left(P_{m}^{1}\right)^{T} A, B P_{m}^{1}, \cdots\right] H(t)\right\} H^{T}(x) F^{2} H(t) .
\end{array}\right\}
$$

This system of matrices in (32) has unknown Haar coefficients A and B. We solve the system simultaneously for unknown with the help of using solution of Lyapunov equation type.

Step 5: Finaly, Haar numerical solutions for $u(x, t)$ and $v(x, t)$ can be constructed from subistitute values of Haar coefficient in (31).

\section{NUMERICAL TEST PROBLEMS}

This section concerning to the numerical test problems and their visualizations.

Problem1. Consider the coupled FPDEs [26]:

$$
\begin{aligned}
& D_{t}^{\alpha} u-u_{x}+v+u=0, \\
& D_{t}^{\beta} v-v_{x}+v+u=0,
\end{aligned}
$$

Subject to initial conditions.

$$
u(x, 0)=\sinh x, v(x, 0)=\cosh x .
$$

The exact solution for $\alpha, \beta=1$ is $u(x, t)=\sinh (x-t), v(x, t)=\cosh (x-t)$, by following the method illustrated in Section 5. (Step 1- Step 4), the above system of linear time FPDEs. In (33) and (34) Corresponding to (31) and (32), when $\alpha=\beta=0.75, m=8$ with substituting initial condition (34) lead to the coupled system:

$$
\begin{aligned}
& H^{T}(x) A P_{8}^{75} H(t)+\sinh (x)-H^{T}(x)\left(P_{8}^{1}\right)^{T} B H(t)+H^{T}(x) B H(t)+H^{T}(x) A H(t)=0, \\
& H^{T}(x) B P_{8}^{.75} H(t)+\cosh (x)-H^{T}(x)\left(P_{m}^{1}\right)^{T} A H(t)+H^{T}(x) B H(t)+H^{T}(x) A H(t)=0 .
\end{aligned}
$$

\begin{tabular}{|c|c|c|c|c|c|c|c|c|c|c|c|c|}
\hline \multirow{2}{*}{$x / t$} & \multicolumn{2}{|c|}{0.0625} & \multicolumn{2}{|c|}{0.1875} & \multicolumn{2}{|c|}{0.3125} & \multicolumn{2}{|c|}{0.4375} & \multicolumn{2}{|c|}{0.5625} & \multicolumn{2}{|c|}{0.6875} \\
\hline & Exact & Appr. & Exact & Appr. & Exact & Appr. & Exact & Appr. & Exact & Appr. & Exact & Appr. \\
\hline 0.0625 & 0.0000 & 0.0312 & 0.1253 & 0.1565 & 0.2526 & 0.2838 & 0.3839 & 0.4151 & 0.5211 & 0.5523 & 0.6665 & 0.6977 \\
\hline 0.1875 & -0.1253 & -0.0941 & 0.0000 & 0.0312 & 0.1253 & 0.1565 & 0.2526 & 0.2838 & 0.3839 & 0.4151 & 0.5211 & 0.5523 \\
\hline 0.3125 & -0.2526 & -0.2214 & -0.1253 & -0.0941 & 0.0000 & 0.0312 & 0.1253 & 0.1565 & 0.2526 & 0.2838 & 0.3839 & 0.4151 \\
\hline 0.4375 & -0.3839 & -0.3527 & -0.2526 & -0.2214 & -0.1253 & -0.0941 & 0.0000 & 0.0312 & 0.1253 & 0.1565 & 0.2526 & 0.2838 \\
\hline 0.5625 & -0.5211 & -0.4899 & -0.3839 & -0.3527 & -0.2526 & -0.2214 & -0.1253 & -0.0941 & 0.0000 & 0.0312 & 0.1253 & 0.1565 \\
\hline 0.6875 & -0.6665 & -0.6353 & -0.5211 & -0.4899 & -0.3839 & -0.3527 & -0.2526 & -0.2214 & -0.1253 & -0.0941 & 0.0000 & 0.0312 \\
\hline
\end{tabular}

The system of (35) has unknown Haar coefficients A and B. We solve this system simultaneously for unknowns. Finely, substitute values of Haar coefficients in (31). Haar solutions of coupled time-(FPDEs.) system are attained; see Figure 2. The obtained Haar solutions are compared with exact solutions at values $(\alpha=0.75)$ and $(\beta=0.75)$ are shown in Table 1 and Table 2.

Table 1. Comparsion between exact and numerical haar solution of $u(x, t)$ of problem 1 
Table 2. Comparsion between exact and numerical haar solution of $v(x, t)$ of problem 1

\begin{tabular}{|c|c|c|c|c|c|c|c|c|c|c|c|c|}
\hline \multirow{2}{*}{$x / t$} & \multicolumn{2}{|c|}{0.0625} & \multicolumn{2}{|c|}{0.1875} & \multicolumn{2}{|c|}{0.3125} & \multicolumn{2}{|c|}{0.4375} & \multicolumn{2}{|c|}{0.5625} & \multicolumn{2}{|c|}{0.6875} \\
\hline & Exact & Appr. & Exact & Appr. & Exact & Appr. & Exact & Appr. & Exact & Appr. & Exact & Appr. \\
\hline 0.0625 & 1.0000 & 1.0312 & 1.0078 & 1.0390 & 1.0314 & 1.0626 & 1.0711 & 1.1023 & 1.1276 & 1.1588 & 1.2018 & 1.2330 \\
\hline 0.1875 & 1.0078 & 1.0390 & 1.0000 & 1.0312 & 1.0078 & 1.0390 & 1.0314 & 1.0626 & 1.0711 & 1.1023 & 1.1276 & 1.1588 \\
\hline 0.3125 & 1.0314 & 1.0626 & 1.0078 & 1.0390 & 1.0000 & 1.0312 & 1.0078 & 1.0390 & 1.0314 & 1.0626 & 1.0711 & 1.1023 \\
\hline 0.4375 & 1.0711 & 1.1023 & 1.0314 & 1.0626 & 1.0078 & 1.0390 & 1.0000 & 1.0312 & 1.0078 & 1.0390 & 1.0314 & 1.0626 \\
\hline 0.5625 & 1.1276 & 1.1588 & 1.0711 & 1.1023 & 1.0314 & 1.0626 & 1.0078 & 1.0390 & 1.0000 & 1.0312 & 1.0078 & 1.0390 \\
\hline 0.6875 & 1.2018 & 1.2330 & 1.1276 & 1.1588 & 1.0711 & 1.1023 & 1.0314 & 1.0626 & 1.0078 & 1.0390 & 1.0000 & 1.0312 \\
\hline
\end{tabular}

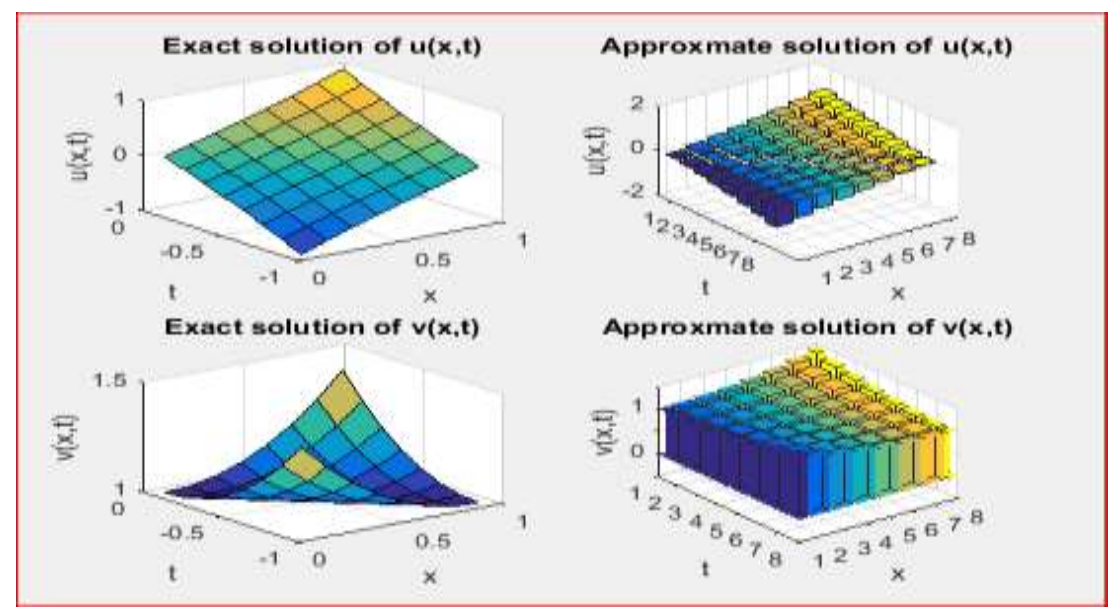

Figure 2. Exact and Haar numerical solutions $u(x, t)$ and $v(x, t)$ of problem 1

Problem 2. Consider the coupled FPDEs. [26]:

$$
\begin{aligned}
& D_{t}^{\alpha} u+u_{x}-2 v=0 \\
& D_{t}^{\beta} v+v_{x}-2 u=0
\end{aligned}
$$

Subject to initial conditions:

$$
u(x, 0)=\sin x, v(x, 0)=\cos x
$$

The exact solution for $\alpha=\beta=1$ is, $u(x, t)=\sin (x+t), v(x, t)=\cos (x+t)$, By following the method illustrated in Section 5. (Step 1- Step 4), the above system of linear time FPDEs. In (36) and (37) Corresponding to (31), when $\alpha=\beta=0.75, m=8$ with substituting initial condition (51) lead to the coupled system:

$$
\begin{aligned}
& H^{T}(x) A P_{8}^{.75} H(t)+\sin (x)+H^{T}(x)\left(P_{8}^{1}\right)^{T} A H(t)-2 H^{T}(x) B H(t)=0, \\
& H^{T}(x) B P_{8}^{.75} H(t)+\cos (x)+H^{T}(x)\left(P_{m}^{1}\right)^{T} B H(t)-2 H^{T}(x) A H(t)=0 .
\end{aligned}
$$

The system of (38) has unknown Haar coefficients A and B. We solve this system simultaneously for unknown. Finely, substitute values of Haar coefficients in (31). Haar solutions of coupled time-(FPDEs.) system are attained; see Figure 3. The obtained Haar solutions are compared with exact solutions at values $(\alpha=0.75)$ and $(\beta=0.75)$ are shown in Table 3 and Table 4. 
Table 3. Comparsion between exact and numerical haar solution of $u(x, t)$ of problem 2

\begin{tabular}{|c|c|c|c|c|c|c|c|c|c|c|c|c|}
\hline \multirow{2}{*}{$x / t$} & \multicolumn{2}{|c|}{0.0625} & \multicolumn{2}{|c|}{0.1875} & \multicolumn{2}{|c|}{0.3125} & \multicolumn{2}{|c|}{0.4375} & \multicolumn{2}{|c|}{0.5625} & \multicolumn{2}{|c|}{0.6875} \\
\hline & Exact & Appr. & Exact & Appr. & Exact & Appr. & Exact & Appr. & Exact & Appr. & Exact & Appr. \\
\hline 0.0625 & 0.1247 & 0.1559 & 0.2474 & 0.2786 & 0.3663 & 0.3975 & 0.4794 & 0.5106 & 0.5851 & 0.6163 & 0.6816 & 0.7128 \\
\hline 0.1875 & 0.2474 & 0.2786 & 0.3663 & 0.3975 & 0.4794 & 0.5106 & 0.5851 & 0.6163 & 0.6816 & 0.7128 & 0.7675 & 0.7987 \\
\hline 0.3125 & 0.3663 & 0.3975 & 0.4794 & 0.5106 & 0.5851 & 0.6163 & 0.6816 & 0.7128 & 0.7675 & 0.7987 & 0.8415 & 0.8727 \\
\hline 0.4375 & 0.4794 & 0.5106 & 0.5851 & 0.6163 & 0.6816 & 0.7128 & 0.7675 & 0.7987 & 0.8415 & 0.8727 & 0.9023 & 0.9335 \\
\hline 0.5625 & 0.5851 & 0.6163 & 0.6816 & 0.7128 & 0.7675 & 0.7987 & 0.8415 & 0.8727 & 0.9023 & 0.9335 & 0.9490 & 0.9802 \\
\hline 0.6875 & 0.6816 & 0.7128 & 0.7675 & 0.7987 & 0.8415 & 0.8727 & 0.9023 & 0.9335 & 0.9490 & 0.9802 & 0.9809 & 1.0121 \\
\hline
\end{tabular}

Table 4. Comparsion between exact and numerical haar solution of $v(x, t)$ of problem 2

\begin{tabular}{|c|c|c|c|c|c|c|c|c|c|c|c|c|}
\hline \multirow{2}{*}{$x / t$} & \multicolumn{2}{|c|}{0.0625} & \multicolumn{2}{|c|}{0.1875} & \multicolumn{2}{|c|}{0.3125} & \multicolumn{2}{|c|}{0.4375} & \multicolumn{2}{|c|}{0.5625} & \multicolumn{2}{|c|}{0.6875} \\
\hline & Exact & Appr. & Exact & Appr. & Exact & Appr. & Exact & Appr. & Exact & Appr. & Exact & Appr. \\
\hline 0.0625 & 0.9922 & 1.0234 & 0.9689 & 1.0001 & 0.9305 & 0.9617 & 0.8776 & 0.9088 & 0.8110 & 0.8422 & 0.7317 & 0.7629 \\
\hline 0.1875 & 0.9689 & 1.0001 & 0.9305 & 0.9617 & 0.8776 & 0.9088 & 0.8110 & 0.8422 & 0.7317 & 0.7629 & 0.6410 & 0.6722 \\
\hline 0.3125 & 0.9305 & 0.9617 & 0.8776 & 0.9088 & 0.8110 & 0.8422 & 0.7317 & 0.7629 & 0.6410 & 0.6722 & 0.5403 & 0.5715 \\
\hline 0.4375 & 0.8776 & 0.9088 & 0.8110 & 0.8422 & 0.7317 & 0.7629 & 0.6410 & 0.6722 & 0.5403 & 0.5715 & 0.4312 & 0.4624 \\
\hline 0.5625 & 0.8110 & 0.8422 & 0.7317 & 0.7629 & 0.6410 & 0.6722 & 0.5403 & 0.5715 & 0.4312 & 0.4624 & 0.3153 & 0.3465 \\
\hline 0.6875 & 0.7317 & 0.7629 & 0.6410 & 0.6722 & 0.5403 & 0.5715 & 0.4312 & 0.4624 & 0.3153 & 0.3465 & 0.1945 & 0.2257 \\
\hline
\end{tabular}

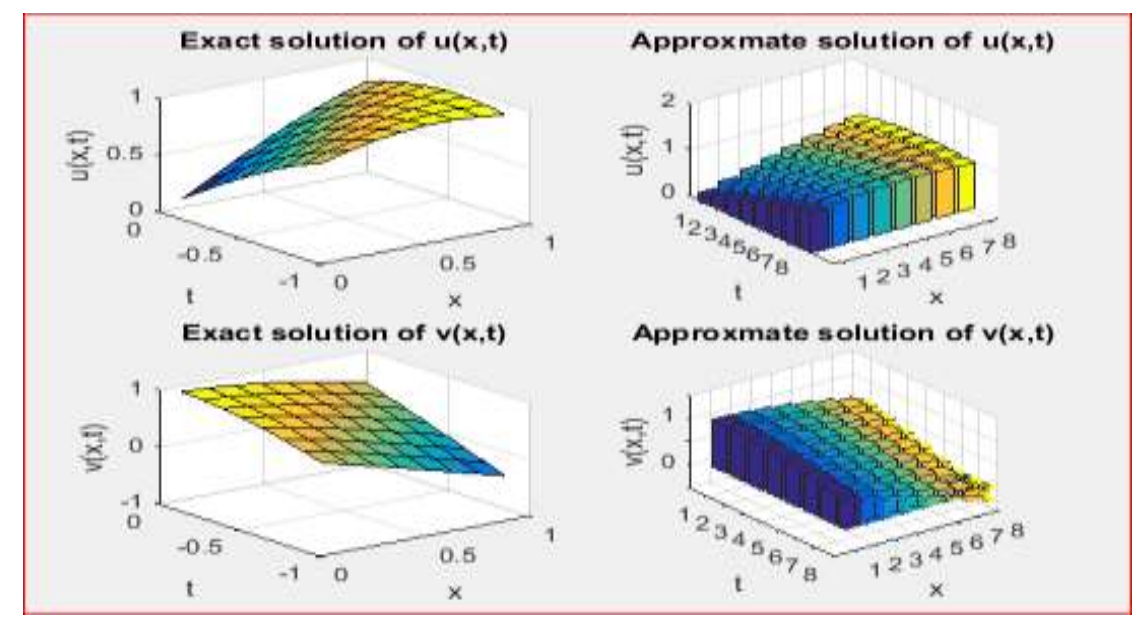

Figure 3. Exact and Haar numerical solutions $u(x, t)$ and $v(x, t)$ of problem 2

\section{CONCLUSION}

In this paper, we applied the Haar operational matrix method of fractional order and integrated with discretization in Caputo's sense to time and spatial derivatives. The main aim of the proposed method is applied to a system of coupled fractional partial differential equations, this approach is used to transform the system into an easily algebraic system of Lyapunov or Sylvester equation type, and the results are compared with the exact solution. The approximate numerical solution via the Haar wavelet method is more elegant in theory, more convenient in numeric computations, and much faster in the data processing.

\section{ACKNOWLEDGEMENTS}

The author would like to thank Al-Mustansiriyah University (www.uomustansiroyah.edu.iq) Baghdad-Iraq for its support in the present work.

\section{REFERENCES}

[1] K. S. Miller and B. Ross, "An introduction to fractional calculus and fractional differential equation," John Wiley, 1993.

[2] I. Podlubny, "Fractional Differential Equations," 198 Academic Press San Diego, California, USA, 1999.

[3] J. sundnes, G. T. Lines and A. Tveito, "Multigrid block conditioning for a coupled system of partial differential equations modeling the electrical activity in the heart," Computer Methods in biome-chanics and biomedical engineering, vol. 5, no. 6, pp. 397-409, 2002. 
[4] J. Sundnes, G. T Lines, A Tveito, "An operator splitting method for solving the bidomain eqations coupled to a volume conductr model for the torso," Mathematical biosciences, vol. 194, no. 2, pp. 233-248, Apr 2005.

[5] W. Shen, "Computer simulation and modeling of physical and biological process using partial differential equation," [Doctoral thesis], University of Kentuky doctorial dissertations, 2007.

[6] R. Katica and S. Hedrish, "Fractional order hybrid system dynamics," Proceeding in applied mathematics and mechanics, vol. 13, no. 1, pp. 25-26, 2013.

[7] Rossikhian, A. Yuriy and M. V. Shitikova, "Application of fractional calculus for dynamic problems of solid mechanics: novael trends and recent result, "Applied Mechanics Reviews, vol. 63, no. 1, pp. 1-52, Jan 2010.

[8] V. Partiban and K. Balachandran, "Solutions of System of Fractional Partial Differential Equations," Applications and Applied Mathematics, vol. 8, no. 1, pp. 289-304, Jun 2013.

[9] K. Fackeldey and R. Krause, "Multiscale coupling in function spaceweak coupling between molecular dynamics and continuum mechanics," International journal for numerical methods in engineering, vol. 79, no. 12, pp. 15171535, Sep. 2009.

[10] A. M. A. El-sayed," Nonlinear fractional differential equations of arbitrary orders," Nonlinear analysis, vol. 33, no. 2, pp. 181-186, 1998.

[11] D. Das and R. K. Bera, "Generalized Differential Transform Method for non-linear Inhomogeneous Time Fractional Partial Differential Equation," International Journal of Sciences \& Applied Research, vol.4, no. 7, pp. 71-77, 2017.

[12] Z. M. Odibat, "A study on the convergence of variational iteration method," Mathematical and Computer Modudeling, vol. 51, no. 9, pp. 1181-1192, 2010.

[13] Y. M. Chen and Y. B. Wu, "Wavelet method for a class of fractional convection-diffusion equation with coeffients," Journal of Computational Science, vol. 1, no. 3, pp. 146-149, 2010.

[14] Y. L. Li and N. Sun, "Numerical solution of fractional differential equations using the generalized block pulse operational matrix," Computers and Mathematics with Application, vol.62, no. 3, pp. 1046-1054, 2011.

[15] Javidi and Ahmad, "Numerical solution of fractional partial differential equations by numerical Laplace inversion technique," Advance in Differential Equations, vol.1, pp. 375-, Dec. 2013.

[16] J. H. He, "A coupling method of a homotopy technique and a perturbation technique for non-linear problem," Int. J. Non-Linear Mech., vol. 35, no. 1, pp. 37-43, 2000.

[17] Ege, S. Muge, and E. Misirli, "A new method for solving nonlinear fractional differential equations," New Trends in Mathematical Sciencse, vol. 5, no. 1, pp. 225-233, 2017.

[18] Z. Odibat, "Approximations of fractional integrals and Caputo fractional derivatives," Applied Mathematics and Computation, vol. 178, no. 2, pp.527-533, 2006.

[19] K. Shah, "Using a numerical method by omitting discretization of data to study numerical solution for for boundary value problems of fractional order differential equations," Mathematical Methods in the Applied Sciences, vol. 42, no. 18, pp. 6944-6959, Dec. 2019.

[20] T. Abdeljawad, R. Amin, K. Shah, Q. Al-Mdallal, and F. Jarad, "Efficient sustainable algorithm for numerical solutions of systems of fractional order differential equations by Haar wavelet collocation method," Aleexandaria Engineering Journal, Mar. 2020.

[21] R. Amin, K. Shah, M. Asif, I. Khan, and F. Ullah, "An efficient algorithm for numerical solution of fractional integro-differential equations via Haar wavelet," Journal of Computational and Mathematics, vol. 1, p. 113028, Jun 2020.

[22] H. Gul, H. Alrabaiah, S. Ali, K. Shah, and S. Muhammed, "Computation of Solution to Fractional Order Partial Reaction Diffusion Equations," Journal of Advanced Research, vol. 15, pp. 1-8, May. 2020.

[23] C. F. Chen and C. H. Hsiao, "Haar wavelet method for solving lumped and distributed-parameter systems," IEEE Proc. Control Theory Appl., vol. 144, no. 1, pp.87-94, 1997.

[24] J. L. Wu, "A wavelet operational method for solving fractional partional differential equation numerically," Applied mathematics and computation, vol. 214, no. 1, pp.31-40, 2009.

[25] S. S. Ray and A. Patra, "Haar wavelet operational methods for the numerical solutions of fractional order nonlinear oscillatory Van der Pol system," Applied mathematics and computayion, vol. 220, pp.659-667, 2013.

[26] H. Jafari, M. Nazari, D. Baleanu, and C.M. Khalique, "A new approach for solving a system of fractional partial differential equations," Computers and Mathematics with Applications, vol. 66, no. 5, pp-838-843, 2013. 